\title{
Modeling of surface texturing in hydrodynamic lubrication
}

\author{
Izhak ETSION* \\ Department of Mechanical Engineering, Technion - Israel Institute of Technology, Haifa 32000, Israel \\ Received: 02 March 2013 / Revised: 25 May 2013 / Accepted: 30 May 2013 \\ (C) The author(s) 2013. This article is published with open access at Springerlink.com
}

\begin{abstract}
Theoretical modeling of surface texturing in hydrodynamic lubrication is a necessary first step to obtain favorable effect of the texturing. This invited review presents a comprehensive summary of the modeling of several basic applications that was done mostly by the author's group at Technion and published in the relevant literature.
\end{abstract}

Keywords: surface texturing; modeling; hydrodynamic lubrication

\section{Introduction}

Surface texturing as a means for enhancing tribological properties of mechanical components is well known for many years. Perhaps the most familiar and earliest commercial application of surface texturing is that of cylinder liner honing. Fundamental research work on various forms and shapes of surface texturing for tribological applications is carried out worldwide and various texturing techniques are employed in these studies. Of all the practical micro-surface patterning methods it seems that laser surface texturing (LST) offers the most promising concept. This is because the laser is extremely fast and allows short processing times; it is clean to the environment and provides excellent control of the shape and size of the texture, which allows realization of optimum designs. Indeed, LST is gaining more and more attention in the tribology community as is evident from the growing number of publications on this subject. LST produces a very large number of micro-dimples on the surface (see Fig. 1) and each of these micro-dimples can serve either as a micro-hydrodynamic bearing in cases of full or mixed lubrication, a micro-reservoir for lubricant in cases of starved lubrication conditions, or

* Corresponding author: Izhak ETSION.

E-mail: etsion@technion.ac.il

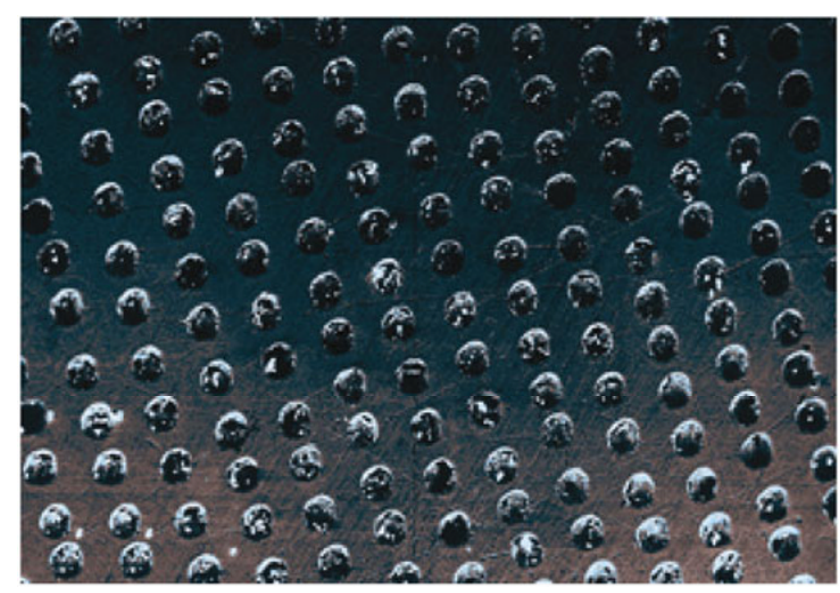

Fig. 1 LST regular micro-surface structure in the form of circular micro-dimples each is having a diameter of the order $100 \mu \mathrm{m}$ and a depth of the order of $1-10 \mu \mathrm{m}$.

a micro-trap for wear debris in either lubricated or dry sliding.

The pioneering work on LST started at Technion in Israel as early as 1996 [1, 2]. At about the same time work on laser surface texturing was done in Germany but unfortunately, most of it is published in the German language and hence, is not even referenced in English archive journals. A few exceptions are papers from the group lead by Geiger at the University of Erlangen-Nuremberg [3,4]. This group used an excimer laser with a mask projection technique. This method was applied to a punch, used in a backward cup extrusion process for the production of rivets, 
and showed a substantial increase of up to $169 \%$ in cold forging tool life. These as well as many other papers on LST are described in a review of the state of the art of LST covering this subject until 2005 [5]. Two other reviews from 2010 focus on laser surface texturing and applications through 2007 [6] and on surface texturing for in-cylinder friction reduction [7]. The evolution of surface texturing, at least from the research aspect, shows a dramatic growth over the last years. Today, the benefits of surface texturing have been successfully demonstrated in numerous tribological applications including automotive, bearings and seals, elasto-hydrodynamic (EHD) lubrication, magnetic storage, etc. While the micro-hydrodynamic bearing function of textured features in cases of full lubrication has been studied extensively, both theoretically and experimentally, and is well understood, the other two functions namely micro-reservoirs for lubricant in cases of starved lubrication conditions, and micro-traps for wear debris are still far from completion, and much research work is still needed in these directions.

In the following, the current state of the art in surface texturing will be described focusing mainly on modeling in hydrodynamic and hydrostatic lubrication applications.

\section{Background}

Surface texturing is a powerful means of enhancing hydrodynamic lubrication between parallel surfaces in relative sliding, which otherwise when un-textured cannot provide any significant hydrodynamic load carrying capacity. This is demonstrated in Fig. 2 showing two parallel surfaces, the lower one moving at a relative sliding velocity $U$ with respect to the upper surface. The surface texturing is represented in Fig. 2(a) by a single protruding asperity attached to the upper surface. The ambient pressure surrounding the two surfaces is $P_{\mathrm{a}}$. In the absence of any texturing the relative sliding velocity results in viscous shear only without any effect on the pressure between the two flat surfaces and, hence, zero load carrying capacity, $F=0$. The introduction of the protruding asperity changes the local film thickness into a converging diverging one, which generates a hydrodynamic

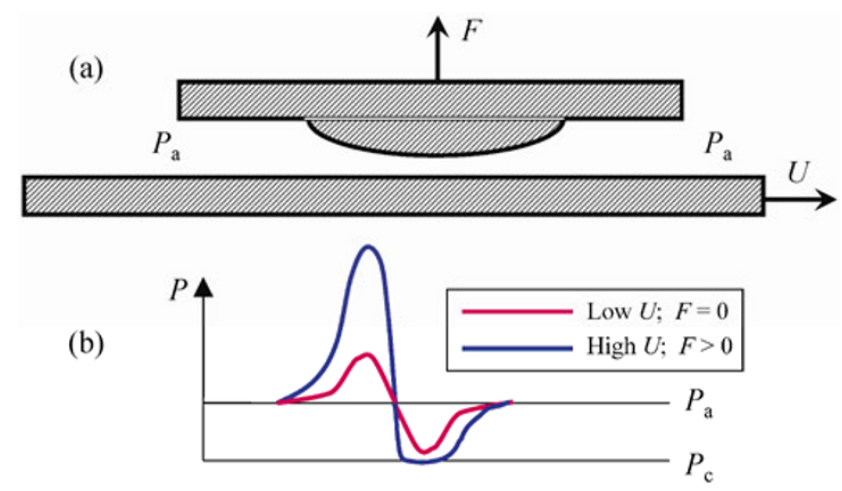

Fig. 2 Schematic description of parallel sliding surfaces: A single protrusion (a), and the hydrodynamic pressure distribution over the single protrusion (b).

pressure distribution as shown in Fig. 2(b). Due to the relative velocity $U$ the pressure increases above $P_{\mathrm{a}}$ in the converging portion and decreases below $P_{\mathrm{a}}$ in the diverging portion of the clearance. At low velocities the maximum pressure is smaller than the absolute value of the cavitation pressure $P_{\mathrm{c}}$ and the pressure distributions is anti-symmetric about $P_{\mathrm{a}}$. Integrating the pressure distribution along its axial span results in zero load capacity since the above and below ambient pressures cancel each other. As the velocity $U$ increases the pressure distribution becomes asymmetric about $P_{\mathrm{a}}$ since the minimum pressure value is bounded from below by the cavitation pressure $P_{\mathrm{c}}$ while the maximum pressure is not limited. The integration of the pressure now results in a net positive value $F>0$. When the textured surface contains a large number of protruding asperities the total load carrying capacity is the sum of their individual contributions. Exactly the same effect with an asymmetric pressure distribution as shown in Fig. 2(b) can be obtained with indented dimples instead of protruding asperities, only in this case the diverging portion of the clearance precedes the converging one. It should be noted here that because of the micro-scale of the asperities or dimples (see Fig. 1) the total load capacity of textured parallel surfaces is relatively small compared to the load capacity that can be generated in conventional hydrodynamic slider bearings. Hence, surface texturing is mostly beneficial in cases where conformal mating surfaces at very small uniform clearances are required, for example, in various sealing applications. Also, as shown in Fig. 3 the dimples configuration is a better 
choice for surface texturing compared to that of protrusions. This is mainly because when the surfaces are brought into contact the real contact area with dimples is much larger than that with protrusions. Hence, the average contact pressure in the dimples case is much smaller and the wear is much lower. Another advantage of the dimples configuration is the smaller separation that can be obtained between the mating surfaces, which allows better sealing and much smaller leakage. Indeed, laser surface texturing has been proven extremely beneficial in various sealing applications for liquids and gas (see Refs. [8-12]). In some of these applications where high pressure liquid has to be sealed, such high pressure may eliminate the cavitation in the individual dimples and thus hamper the generation of hydrodynamic load carrying capacity. The solution for this problem is a partial surface texturing adjacent to the high pressure side as shown in Fig. 4 . When the clearance between two parallel surfaces has a step change (see Fig. 4(a)) and the larger clearance $h_{\max }$ is facing the higher pressure $p_{0}$, a hydrostatic load carrying capacity can be generated due to the restriction in the Poiseuille flow caused by the smaller clearance $c$ facing the lower pressure $p_{\mathrm{a}}$. A similar effect is obtained by "partial" surface texturing where high density dimples at the high pressure end form an equivalent "step" of height $h_{\mathrm{eq}}$ as shown in Fig. 4(b). This solution has been used successfully in high pressure seals as described in Ref. [13]. Both the original "full" texturing and its modification form of "partial" texturing, as well as textured micro-grooves, were successfully applied to piston rings and cylinder liners [14-17]. The partial texturing concept was also found very useful in generating substantial hydrodynamic load capacity in hydrodynamic slider bearings and thrust bearings of the simplest form of parallel sliding disks $[18,19]$. Here the effect of the equivalent step is similar to the very efficient Raleigh step in a slider bearing. Through extensive theoretical modeling it was found that the most important parameter in surface texturing for full fluid film lubrication is the aspect ratio of the dimples (depth over diameter ratio). It is this parameter that can be optimized in order to provide maximum load carrying capacity, maximum film stiffness and minimum friction coefficient. The area density, $S_{\mathrm{p}}$, of the texturing also affects the efficiency of surface texturing and this parameter also can be optimized. Yet another important parameter that can be optimized in cases of partial texturing is the textured portion ratio $\alpha=B_{\mathrm{p}} / B$ (see Figs. 5(a) and 5(b)).
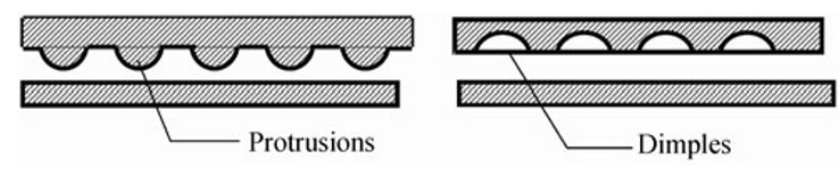

- Complicated etching technology

- Simple \& cheap laser technology

- High wear

- Low wear

- High leakage (seals)

- Low leakage (spacing)

Fig. 3 Advantages of a dimples configuration in surface texturing compared to that of protrusions.

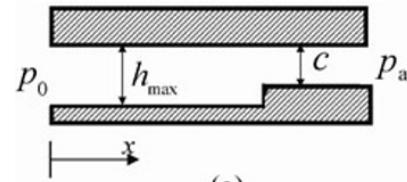

(a)

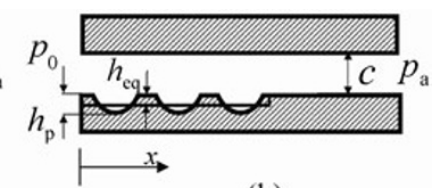

(b)
Fig. 4 A comparison of two equivalent sets of parallel surfaces: A step change in the clearance (a), and partial surface texturing (b).

(a)

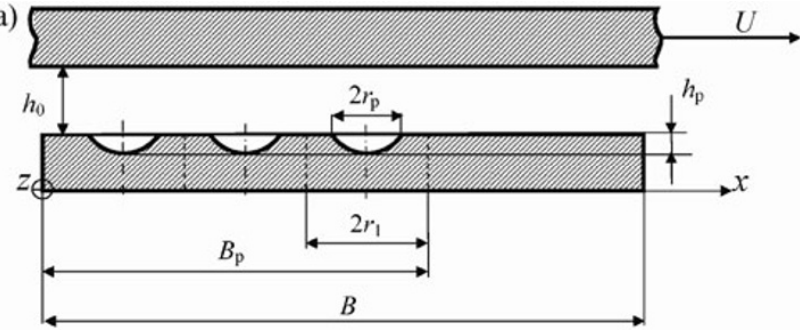

(b)

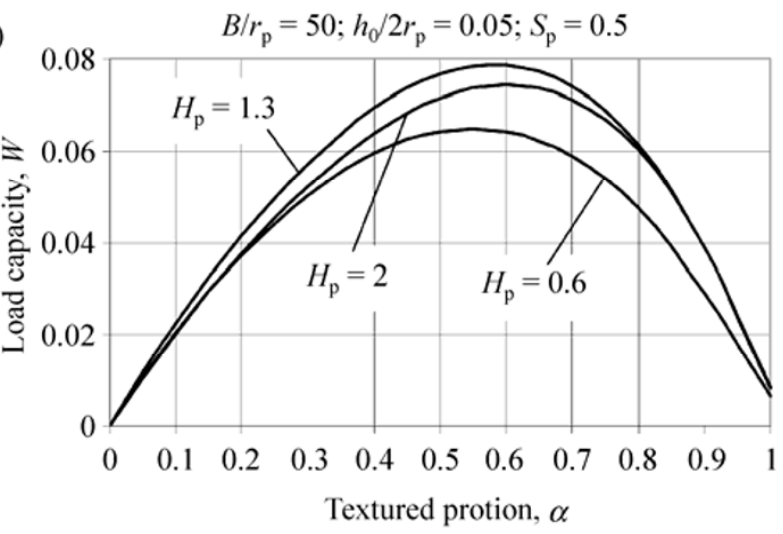

Fig. 5 (a) A cross section of a partially laser surface textured parallel slider bearing. (b) The effect of the textured portion, $\alpha=$ $B_{\mathrm{p}} / B$, on the dimensionless load carrying capacity of an infinitely long parallel slider at various dimensionless dimple depths, $H_{\mathrm{p}}=$ $h_{\mathrm{p}} / h_{0}($ see (a)). 


\section{Basic modeling for different applications}

\subsection{Mechanical seals}

We shall use a typical textured seal application [8] to demonstrate the basic modeling of surfaces texturing for mechanical seals. The geometrical model of the textured surface is displayed in Figs. 6 and 7. Each dimple is modeled by an axi-symmetric spherical segment with a base radius $r_{\mathrm{p}}$ and depth $h_{\mathrm{p}}$ (see Fig. 7). The dimples are distributed uniformly over the annular surface with an area density $S_{p}$. Each dimple is located in the center of an imaginary square cell of sides $2 r_{1} \times 2 r_{1}$ (see Fig. 6(c)) where

$$
r_{1}=\frac{r_{\mathrm{p}}}{2} \sqrt{\frac{\pi}{S_{\mathrm{p}}}}
$$

It is assumed that the clearance between the nominally parallel mating surfaces (see Fig. 7) is fully filled with an incompressible viscous (Newtonian) fluid having a constant viscosity $\mu$. The ratio $R_{\mathrm{i}}$ between

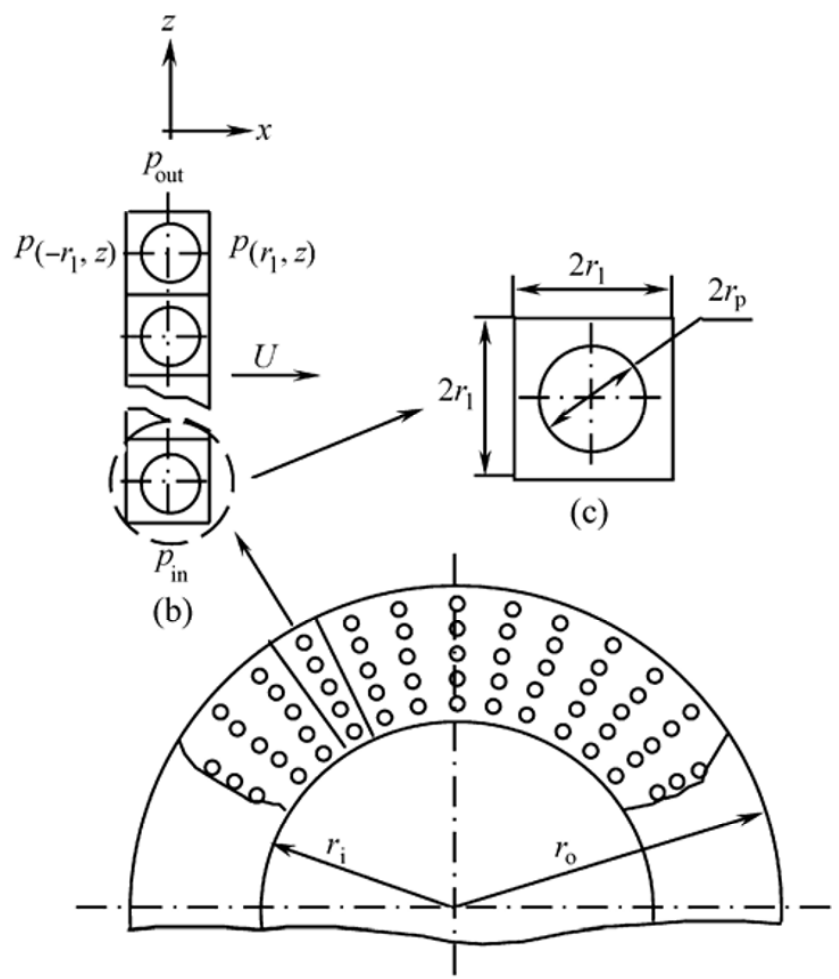

(a)

Fig. 6 Schematic description of a textured seal ring: (a) dimples distribution; (b) a single dimples column with its coordinate system and boundary conditions; (c) individual dimple cell.

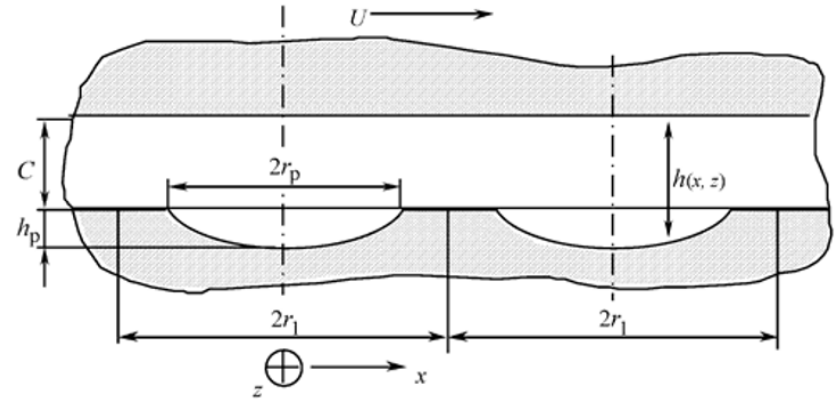

Fig. 7 Film thickness and geometry of dimples in a textured seal.

the inner and outer radii, $r_{\mathrm{i}}$ and $r_{\mathrm{o}}$ of the seal ring under consideration is larger than 0.7. This allows to neglect curvature effects and consequently, a circular sector containing one dimples column in the radial direction (see Figs. 6(a) and 6(b)) is assumed to be rectangular, subjected in the lateral $x$ direction to a relative sliding velocity $U$, corresponding to the tangential velocity at the mean radius of the seal ring.

The two-dimensional, steady state form of the Reynolds equation for an incompressible Newtonian fluid in a laminar flow is given by

$$
\frac{\partial}{\partial x}\left(h^{3} \frac{\partial p}{\partial x}\right)+\frac{\partial}{\partial z}\left(h^{3} \frac{\partial p}{\partial z}\right)=6 \mu U \frac{\partial h}{\partial x}
$$

where $z$ and $x$ are the radial and circumferential directions Cartesian coordinates, respectively, $h$ and $p$ are the local film thickness and pressure, respectively, at a specific point of the seal. In order to reduce Eq. (2) to a dimensionless form the dimensionless Cartesian coordinates $X$ and $Z$, dimensionless local film thickness $H$ and dimensionless pressure $P$ are defined as:

$$
X=\frac{x}{r_{\mathrm{p}}} ; \quad Z=\frac{z}{r_{\mathrm{p}}} ; \quad H=\frac{h}{C} ; \quad P=\frac{p}{p_{\mathrm{a}}}
$$

where $p_{\mathrm{a}}$ is the ambient pressure and $C$ is the seal clearance. After substitution of Eq. (3) into Eq. (2) the Reynolds equation in its dimensionless form becomes

$$
\frac{\partial}{\partial X}\left(H^{3} \frac{\partial P}{\partial X}\right)+\frac{\partial}{\partial Z}\left(H^{3} \frac{\partial P}{\partial Z}\right)=\Lambda \frac{\partial H}{\partial X}
$$

where the dimensionless parameter $\Lambda$ is given by

$$
\Lambda=\frac{6 \mu U r_{\mathrm{p}}}{p_{\mathrm{a}} C^{2}}
$$


The dimensionless local film thickness $H$ over one imaginary dimple cell as a function of the local dimensionless Cartesian coordinates $\bar{X}$ and $\bar{Z}$, measured from the center of the imaginary dimple cell is given by

$$
\begin{aligned}
& H(\bar{X}, \bar{Y}) \\
& = \begin{cases}1 & \text { for } \bar{X}^{2}+\bar{Z}^{2}>1 \\
1+\sqrt{\left(\frac{\varepsilon}{2 \delta}+\frac{1}{8 \varepsilon \delta}\right)^{2}-\left(\bar{X}^{2}+\bar{Z}^{2}\right) \frac{1}{4 \delta^{2}}} & \\
-\left(\frac{1}{8 \varepsilon \delta}-\frac{\varepsilon}{2 \delta}\right) & \text { for } \bar{X}^{2}+\bar{Z}^{2}<1\end{cases}
\end{aligned}
$$

where $\varepsilon=h_{\mathrm{p}} /\left(2 r_{\mathrm{p}}\right)$ is the dimple's aspect ratio, $\delta$ is the dimensionless seal clearance $C /\left(2 r_{\mathrm{p}}\right)$ and the axes of $\bar{X}$ and $\bar{Z}$ (not shown in Fig. 6) are parallel to these of $x$ and $z$, respectively.

By specifying $\varepsilon$ and $\delta$ and hence, the film thickness distribution $H(x, z)$, the dimensionless parameter $\Lambda$, and the relevant boundary conditions, Eq. (4) can be solved for the pressure distribution in the seal clearance. Integrating the pressure over the seal area gives the opening force acting in the axial direction to prevent contact between the rings for reliable operation of the mechanical seal.

Since the micro-dimples are evenly distributed it is assumed that the pressure distribution is periodic in the circumferential direction with a period equal to the imaginary square cell size $2 r_{1}$. Because of this it is sufficient to consider only one radial dimples column as shown in Fig. 6(b), with the following boundary conditions:

$$
p\left(x, z=r_{\mathrm{i}}\right)=p_{\text {in }} ; \quad p\left(x, z=r_{\mathrm{o}}\right)=p_{\text {out }}
$$

Dimensionless values of the pressure at the two edges of one dimples column are obtained by normalization according to Eq. (3). Periodicity condition of the pressure is applied in the circumferential direction so that

$$
p\left(x=-r_{1}, z\right)=p\left(x=r_{1}, z\right)
$$

The boundary conditions in dimensionless form are given as follows:

$$
P\left(X, Z=\frac{r_{\mathrm{i}}}{r_{\mathrm{p}}}\right)=\frac{p_{\text {in }}}{p_{\mathrm{a}}} ; \quad P\left(X, Z=\frac{r_{\mathrm{o}}}{r_{\mathrm{p}}}\right)=\frac{p_{\text {out }}}{p_{\mathrm{a}}} ;
$$

$$
P\left(X=-\frac{r_{1}}{r_{\mathrm{p}}}, Z\right)=P\left(X=\frac{r_{1}}{r_{\mathrm{p}}}, Z\right)
$$

The boundary conditions at the inner and outer radii of the seal, Eq. (7), and the periodicity condition, Eq. (8), should be complemented by the conditions at the boundaries of possible cavitation regions associated with each individual dimple. As explained in Section 2 above, these cavitation regions are responsible for the asymmetric hydrodynamic pressure distribution and, hence, are the only source for load carrying capacity in parallel surface sliding. The relatively simple Reynolds boundary condition, also known as the Swift Stieber condition (see Ref. [20]), or any other more advanced cavitation boundary conditions (e.g., Ref. [21]), can be assumed. The Reynolds condition implies that on the cavitation boundary the pressure gradient normal to the boundary is zero and the pressure inside the cavitation region is retained constant close to zero.

The Reynolds equation, Eq. (4), with its appropriate boundary conditions can be solved by a finite difference method using a non-uniform grid over the radial dimples column, shown in Fig. 6(b), where a denser grid is applied within the dimple areas. Numerical tests show that, for dimple density values $S_{\mathrm{p}}$ in the range between $10 \%$ to $50 \%$, the best accuracy of pressure calculation is obtained when the grid applied in the area of the dimples is about five times denser than that outside of the dimples.

The finite difference method leads to a set of linear algebraic equations for the nodal values of the pressure which should be solved with the boundary conditions at the inner and outer radii of the seal, Eq. (7), and the periodicity condition, Eq. (8). This linear equations set may be solved by various standard methods. The successive over-relaxation Gauss-Seidel iterative method [22] is one possible method. It requires an initial approximation of the solution and, in the case of a mechanical seal, for example, a linear hydrostatic pressure distribution may be used for this purpose if a more precise solution is unknown. Although the iteration algorithm of Gauss-Seidel is not always the most effective one, this method is convenient for the determination of the previously unknown cavitation regions. 
The basic modeling described above for mechanical seals can be easily modified to fit different applications. These modifications include, for example, solving a non-linear Reynolds equation for compressible fluids [11], solving the Reynolds equation simultaneously with a dynamic equation for piston rings [14] or with the equation of elasticity for elastomeric seals and bearings [23, 24]. In the following few of these modifications are presented in more details.

\subsection{Gas seals}

The non-linear Reynolds equation for compressible fluid applications such as gas seals [11] or gas bearings has the form:

$$
\frac{\partial}{\partial x}\left(p h^{3} \frac{\partial p}{\partial x}\right)+\frac{\partial}{\partial z}\left(p h^{3} \frac{\partial p}{\partial z}\right)=6 \mu U \frac{\partial}{\partial x}(p h)
$$

After substitution of Eq. (2) into Eq. (10) the Reynolds equation in its dimensionless form becomes

$$
\frac{\partial}{\partial X}\left(P H^{3} \frac{\partial P}{\partial X}\right)+\frac{\partial}{\partial Z}\left(P H^{3} \frac{\partial P}{\partial Z}\right)=\frac{\lambda}{\delta^{2}} \frac{\partial}{\partial X}(P H)
$$

where $\lambda=3 \mu U / 2 r_{\mathrm{p}} p_{\mathrm{a}}$ is the dimensionless seal parameter and $\delta=c / 2 r_{\mathrm{p}}$ is the dimensionless seal clearance.

A finite element method using a non-uniform grid over the radial dimples' column, shown in Fig. 6(b), can be used to solve the Reynolds equation, Eq. (11), with its appropriate boundary conditions, where a dense grid is applied within the dimple areas. Note that for the case of compressible fluids there is no cavitation to deal with, which somewhat simplifies the boundary conditions. A variational Galerkin formulation can be utilized in order to apply the finite element method to the Reynolds differential equation [25]

$$
\begin{aligned}
\iint_{A} & {\left[\frac{\partial}{\partial X}\left(P H^{3} \frac{\partial P}{\partial X}\right)+\frac{\partial}{\partial Z}\left(P H^{3} \frac{\partial P}{\partial Z}\right)-\frac{\lambda}{\delta^{2}} \frac{\partial}{\partial X} H\right] } \\
\cdot & N_{i}(X) N_{j}(Z) \mathrm{d} X \mathrm{~d} Z=0
\end{aligned}
$$

where $A$ is the area of one dimples' column, $N_{i}(X)$ and $N_{j}(Z)$ are Lagrange polynomials and all their possible products $N_{i}(X) N_{j}(Z)$ are the weight functions. The solution $P(X, Z)$ is approximated by the double series

$$
P(X, Z)=\sum_{i, j} P_{i j} N_{i}(X) N_{j}(Z)
$$

where $P_{i j}$ are the approximate pressure values at the nodal points of the finite element grid. Note that the Lagrange polynomials used for the approximation of the pressure distribution in the seal are the same as these of the shape functions. The finite element method leads to a set of nonlinear algebraic equations for the nodal values of the pressure that should be solved with the boundary conditions. This set of equations can be solved by the Newton gradient method. It requires an initial approximation of the solution, which can be a linear hydrostatic pressure distribution if a more precise solution is unknown. Although the convergence of the Newton gradient method is sensitive to the initial approximation, the convergence rate, in the case of successful initial approximation, is very high.

\subsection{Parallel thrust bearings}

A schematic representation of a parallel thrust bearing [18] is shown in Fig. 8. A plain disk (D) is rotating relative to a number of identical stationary pads $(\mathrm{P})$. Each pad, when properly textured, develops the same hydrodynamic force. Hence, in order to evaluate the load carrying capacity of the complete parallel thrust

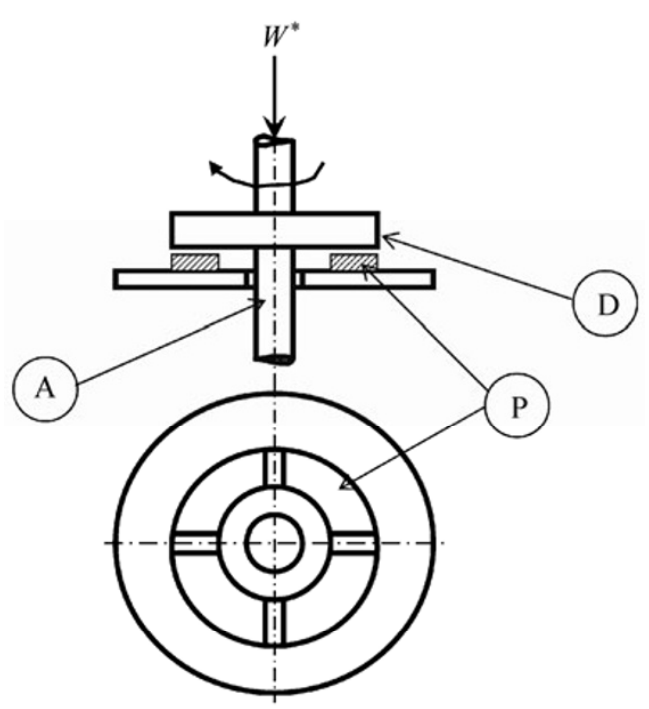

A-axis of rotation; D-rotating disk; P-pad.

Fig. 8 Schematic of a parallel thrust bearing. 
bearing it is sufficient to determine the hydrodynamic pressure distribution over a single pad. A simplified geometrical model of a single pad in the form of a rectangular parallel slider is displayed in Fig. 5(a). The dimples are regularly distributed over a portion, $0 \leqslant \alpha \leqslant 1$ (where $\alpha=B_{\mathrm{p}} / B$ ), of the slider width, $B$, in the sliding direction, $x$, and over the full slider length, $L$, along the direction $z$ (not shown in Fig. 5(a)).

In order to reduce Eq. (2) to a dimensionless form, the dimensionless coordinates $X$ and $Z$, dimensionless local film thickness, $H$, and dimensionless local pressure, $P$, are defined as follows

$$
X=\frac{x}{r_{\mathrm{p}}} ; Z=\frac{z}{r_{\mathrm{p}}} ; H=\frac{h}{h_{0}} ; P=\frac{1}{\Lambda}\left(\frac{p}{p_{\mathrm{a}}}-1\right)
$$

where $p_{\mathrm{a}}$ is the ambient pressure, $h_{0}$ is the bearing clearance (see Fig. 5(a)) and $\Lambda$ is the dimensionless bearing number, given as

$$
\Lambda=\frac{3 \mu U}{2 r_{\mathrm{p}} p_{\mathrm{a}}}
$$

Substituting Eqs. (14) and (15) into Eq. (2) yields the Reynolds equation in its dimensionless form:

$$
\frac{\partial}{\partial X}\left(H^{3} \frac{\partial P}{\partial X}\right)+\frac{\partial}{\partial Z}\left(H^{3} \frac{\partial P}{\partial Z}\right)=\frac{1}{\delta^{2}} \frac{\partial H}{\partial X}
$$

where $\delta$ is the dimensionless clearance, defined as $\delta=h_{0} / 2 r_{\mathrm{p}}$. The pressure along the slider boundaries is equal to the ambient pressure $p_{\mathrm{a}}$ that, by the definitions in Eq. (14), corresponds to zero dimensionless pressure.

The analytical model is valid for all values of slider length, $L$, and width, $B$. However, if the slider is long enough in the $z$ direction (normal to the sliding velocity), with a ratio $L / B>4$, the end effects in this direction can be neglected. In this special case the pressure distribution is periodical in the $z$ direction with a period equal to the imaginary cell size $2 r_{1}$. Hence, because of this periodicity, it is sufficient to consider a single column of dimples along the $x$ direction. Due to symmetry of the dimples column about its $x$ axis, the pressure distribution will be also symmetric about this axis. Therefore, for the complete pressure distribution it is sufficient to consider only one half of the dimples column with $z$ varying from 0 to $r_{1}$. From the periodicity, symmetry and continuity of the pressure distribution, it follows that:

$$
\frac{\partial P}{\partial Z}(X, 0)=\frac{\partial P}{\partial Z}\left(X, r_{1}\right)=0
$$

The dimensionless pressure obtained from a solution of the Reynolds equation Eq. (16) with its appropriate boundary conditions, for a finite or for an infinitely long slider is numerically integrated over the slider area yielding the dimensionless load carrying capacity $\bar{W}$, which is related to the dimensional load carrying capacity $W^{*}$ in the form:

$$
\bar{W}=\frac{2 W^{*}}{3 \mu U r_{\mathrm{p}}}
$$

\subsection{Piston rings}

Figure 9 shows a piston ring segment [26] with partial LST applied at the middle portion of the ring width. However, the textured portion can be located elsewhere as shown schematically in Fig. 10. Here $w^{*}$ is the piston ring width, $b_{\mathrm{p}}$ is the axial length of the textured zone, $x$ is the axial direction of the cylinder liner, and $z$ is the circumferential direction of the piston ring. In Fig. 10(a) the textured zone is symmetrically located at the center of the ring; in Fig. 10 (b) it is located symmetrically at both ends of the ring and in Fig. 10(c) at an arbitrary distance $d$ from the ring center.

A detailed description of a partial central symmetrical texturing is presented in Fig. 11. The dimples are uniformly distributed, with an area density $S_{\mathrm{p}}$ within the strip $b_{\mathrm{p}}$ of the piston ring full width $w^{*}$. The textured zone is bounded with two un-textured

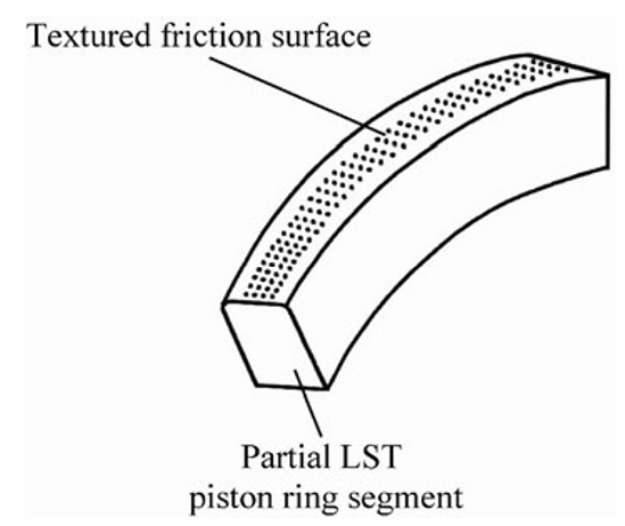

Fig. 9 A segment of partially textured piston ring. 

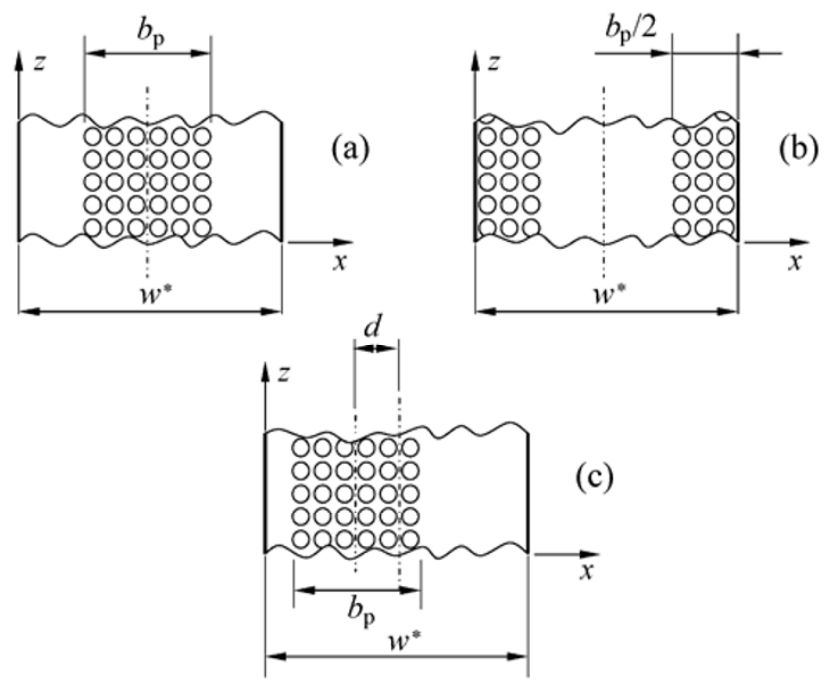

Fig. 10 Different locations of the textured zone: (a) symmetrically in the center; (b) symmetrically at both ends; (c) arbitrarily at a distance $d$ from the ring center [26].

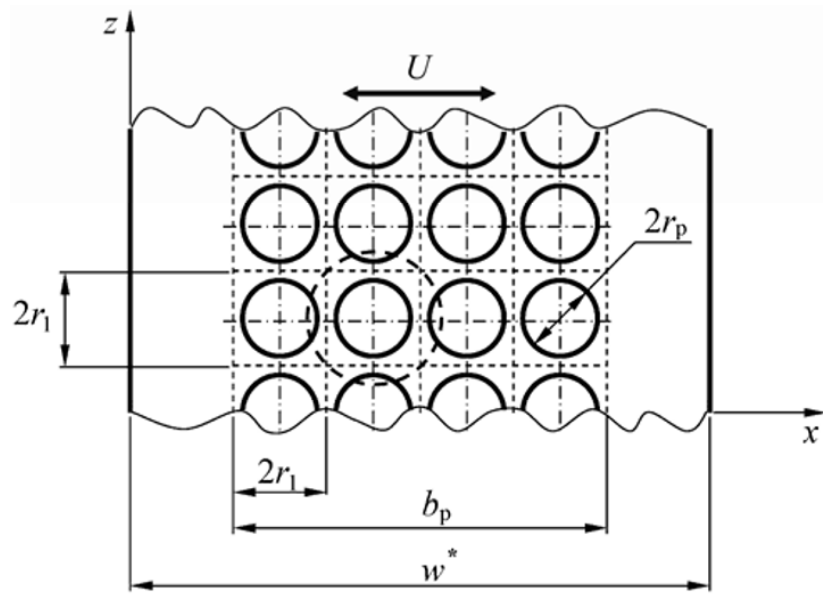

Fig. 11 A geometrical model of central symmetrical textured surface of a piston ring [26].

strips of width $\left(w^{*}-b_{\mathrm{p}}\right) / 2$ on each of its side. Because of the periodicity of the surface texturing in the circumferential direction $z$, and the symmetry in each axial dimple column of width $2 r_{1}$ about its longitudinal axis, it is sufficient to consider the pressure distribution within just one half of one dimple column.

Assuming a slider-crank mechanism that drives the piston [14], the sliding velocity $U$ is time dependent and the clearance between ring and cylinder liner varies with time during each cycle of the ring reciprocal motion. Hence, a simultaneous solution of the Reynolds equation with squeeze effect and the equation of piston ring radial motion is required. These equations are given in the form:

$$
\begin{gathered}
\frac{\partial}{\partial x}\left(h^{3} \frac{\partial p}{\partial x}\right)+\frac{\partial}{\partial z}\left(h^{3} \frac{\partial p}{\partial z}\right)=6 \mu U \frac{\partial h}{\partial x}+12 \mu \frac{\partial h}{\partial t} \\
\rho h_{\mathrm{r}} \frac{\partial^{2} c}{\partial t^{2}}=p_{\mathrm{h}}-p_{\mathrm{e}}
\end{gathered}
$$

where $h$ is the instantaneous local film thickness at a specific point $(x, z)$ (see Fig. 12), $p$ is the instantaneous local hydrodynamic pressure, $\mu$ is the dynamic viscosity of the fluid, $c(t)$ is the instantaneous nominal clearance, $\rho$ and $h_{\mathrm{r}}$ are the piston ring density and height, respectively, $p_{\mathrm{e}}$ is the total external pressure on the ring consisting of gas pressure and piston ring elasticity, and $p_{\mathrm{h}}$ is the instantaneous average hydrodynamic pressure between the ring and liner.

A simultaneous solution of Eqs. (19) and (20) provides the time behavior of both the clearance and pressure between the piston ring and cylinder liner surfaces. The dimensionless form of the differential Eqs. (19) and (20) is given by

$$
\begin{gathered}
\frac{\partial}{\partial X}\left(H^{3} \frac{\partial P}{\partial X}\right)+\frac{\partial}{\partial Z}\left(H^{3} \frac{\partial P}{\partial Z}\right)=2 \Lambda_{1} \Phi(\tau) \frac{\partial H}{\partial X}+12 \frac{\partial H}{\partial \tau} \\
\Lambda_{2} \frac{\partial^{2} C}{\partial \tau^{2}}=P_{\mathrm{h}}-P_{\mathrm{e}}
\end{gathered}
$$

where $X$ and $Z$ are the dimensionless Cartesian coordinates, $H$ is the dimensionless instantaneous local film thickness, $\Phi(\tau)$ is a trigonometric function governing the kinematics of crank mechanism (see Ref. [14]), and $P, P_{\mathrm{h}}$ and $P_{\mathrm{e}}$ are the corresponding

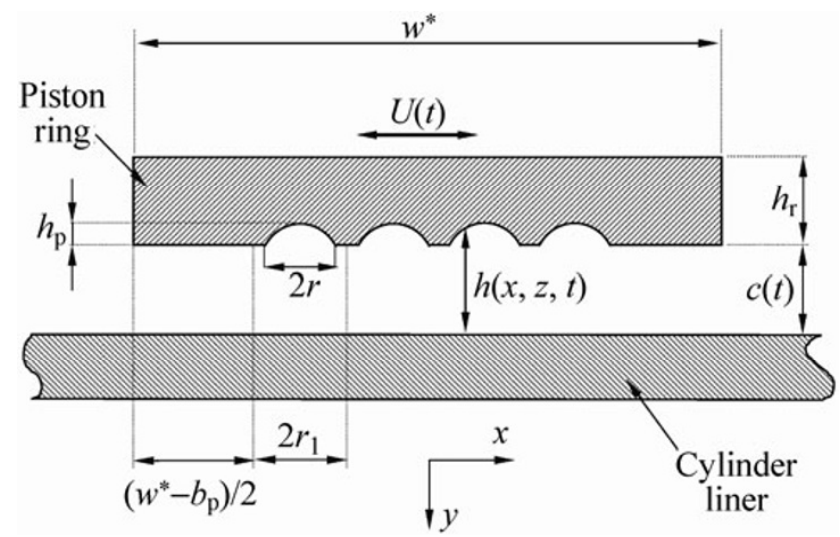

Fig. 12 Piston ring, cylinder liner and film thickness cross-section [26]. 
dimensionless pressures. These various parameters are normalized in the form:

$$
X=\frac{x}{r_{\mathrm{c}}} ; \quad Z=\frac{z}{r_{\mathrm{c}}} ; \quad H=\frac{h}{r_{\mathrm{c}}} ; \quad P=\frac{p-p_{\mathrm{a}}}{p_{\mathrm{a}}}
$$

Note that $r_{\mathrm{c}}$ the crank radius is half the piston stroke, and $p_{\mathrm{a}}$ is an ambient pressure.

The other dimensionless parameters, $\Lambda_{1}, \delta, \varepsilon_{\mathrm{c}}$ and $\Lambda_{2}$ are given by

$$
\Lambda_{1}=\frac{3 \mu \omega}{p_{\mathrm{a}}} ; \quad \delta=\frac{2 r_{\mathrm{p}}}{r_{\mathrm{c}}} ; \quad \varepsilon_{\mathrm{c}}=\frac{h_{\mathrm{p}}}{r_{\mathrm{c}}} ; \quad \Lambda_{2}=\frac{\rho h_{\mathrm{r}} r_{\mathrm{c}} p_{\mathrm{a}}}{\mu^{2}}
$$

More details as well as the formulation of the friction force between the piston ring and cylinder liner, due to viscous shear stresses can be found in Ref. [26].

\subsection{Soft elasto-hydrodynamic lubrication (SEHL)}

Theoretical modeling of surface texturing for applications involving elastomeric components such as O-rings and various other geometries of reciprocating sealing rings, lip seals, elastomeric bearings etc. is relatively new [23, 24]. Hence, differently from the previous applications, no experimental evidence has yet been accumulated to validate these models. There are two options in SEHL applications regarding the surface to be textured. Texturing can be applied either on the rigid surface or on the soft elastomeric one. While texturing elastomeric surfaces may present a technological challenge it has one obvious advantage at least in reciprocating sealing where the area to be textured is much smaller if the short elastomeric seal rather than the long reciprocating shaft is textured. Another advantage of texturing the soft rather than the rigid surface may be the prevention of wear of the soft elastomer by the harder surface features (protrusions or indentations) during unfavorable lubrication conditions. In this section the modeling of a textured elastomeric sleeve in rotary sliding [24] is described as an example for SEHL application.

A schematic illustration of the SEHL model is shown in Fig. 13(a). A stationary elastomer sleeve with rectangular cross-section is fitted on a rotating shaft. A regular surface texturing is applied to the inner surface of the sleeve as shown schematically in Fig. 13(b) and in more details in Fig. 13(c). The dimples are
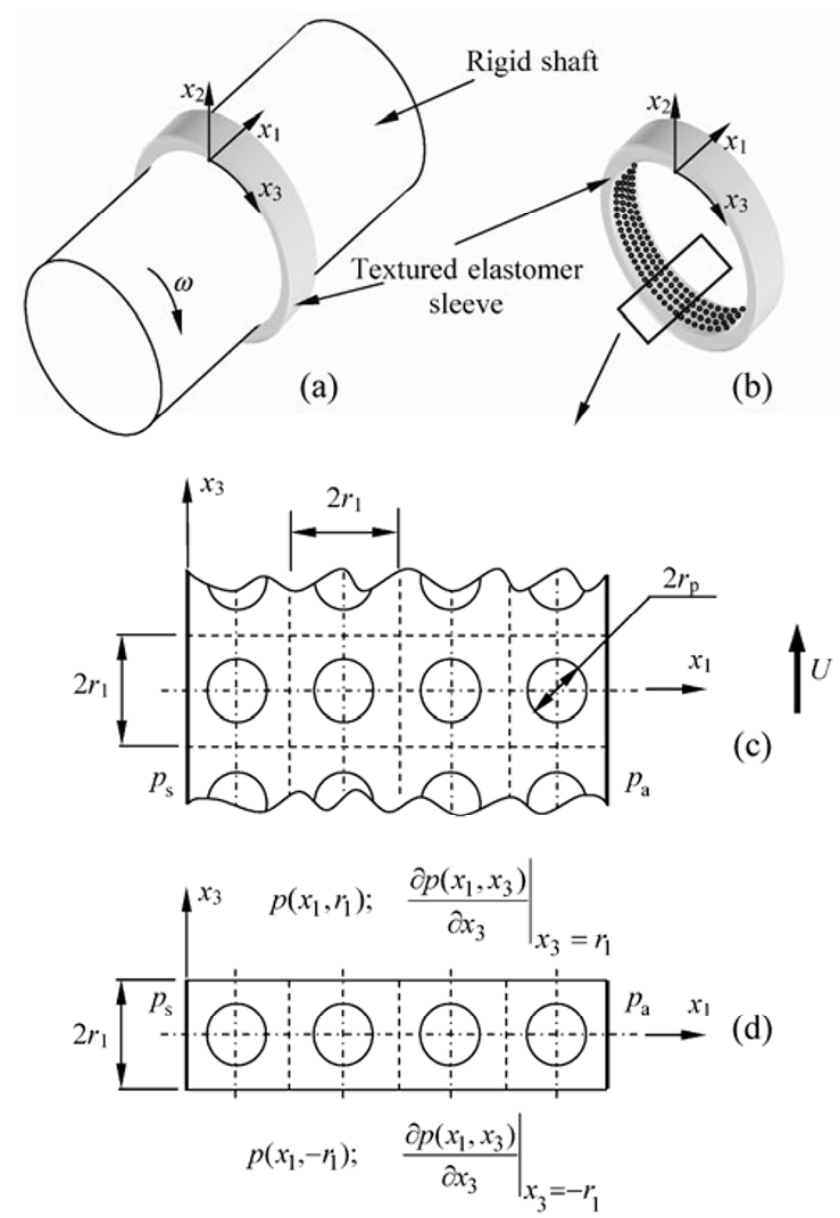

Fig. 13 (a) A schematic of the SEHL model; (b) an elastomer sleeve with regular surface texturing; (c) a geometrical model of a textured surface; (d) a single column of dimples [24].

uniformly distributed on the elastomer surface with an area density $S_{\mathrm{p}}$. The dimples are arranged in a grid of longitudinal columns (see Fig. 13(d) for a single column) and circumferential rows. As in all previous applications here too all the dimples are identical spherical segments with a base radius, $r_{\mathrm{p}}$, and a maximum depth, $h_{\mathrm{p}}$. Each dimple is located in the center of an imaginary square cell of sides $2 r_{1} \times 2 r_{1}$.

The longitudinal cross-section of the elastomer sleeve before (dashed line) and after (solid line) deformation is shown in Fig. 14(a). The sleeve is fitted on the shaft with an interference fit, $c$, while its outer circumference is fixed to a rigid foundation. Due to the surface texturing a hydrodynamic pressure is generated between the rotating shaft and the elastomer sleeve, causing deformation of the latter. This pressure may be sufficient to separate the initially interfering mating 


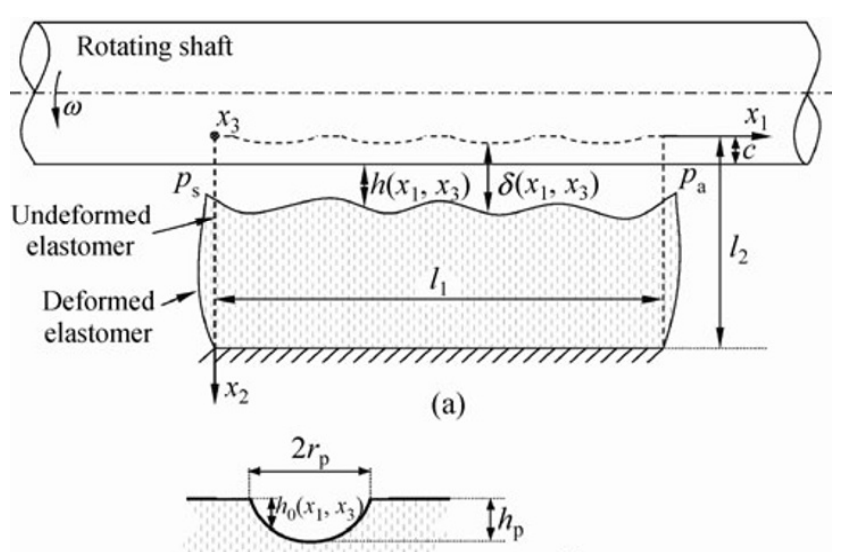

(b)

Fig. 14 (a) A schematic cross-section (not to scale) of the elastomer sleeve; (b) a cross-section through a single undeformed dimple [24].

surfaces by a thin fluid film with a thickness distribution $h\left(x_{1}, x_{3}\right)$, which is given by

$$
h\left(x_{1}, x_{3}\right)=-c+h_{0}\left(x_{1}, x_{3}\right)+\delta\left(x_{1}, x_{3}\right)
$$

In Eq. (25) $h_{0}\left(x_{1}, x_{3}\right)$ is the local depth of an undeformed dimple (see Fig. 14(b)), and $\delta\left(x_{1}, x_{3}\right)$ is the variation of the local film thickness due to radial deformation of the elastomer inner surface.

For analyzing the surface texturing effect a full hydrodynamic lubrication between the textured elastomer and rigid shaft is assumed during operation, i.e., no contact between the mating surfaces is allowed. The fluid film thickness is assumed much smaller than the shaft radius, thus curvature in the circumferential direction $x_{3}$ can be neglected. This allows using Cartesian instead of cylindrical coordinates and replacing rotational by translational velocity in the $x_{3}$ direction. Roughness of the shaft and the elastomer surfaces is neglected except for the regular surface texturing. The fluid is assumed Newtonian, incompressible, and with a constant viscosity $\mu$. Further assumptions related to relevant properties of the fluid and the elastomer can be found in Ref. [24].

Based on these assumptions the Reynolds equation for the local pressure in the fluid film, and the set of elasticity equations for the elastomer deformation are given in the form (see Ref. [23]):

$$
\frac{\partial}{\partial x_{1}}\left(h^{3} \frac{\partial p}{\partial x_{1}}\right)+\frac{\partial}{\partial x_{3}}\left(h^{3} \frac{\partial p}{\partial x_{3}}\right)=6 \mu U \frac{\partial h}{\partial x_{3}}
$$

$$
\left[\frac{1}{2}\left(u_{i, j}^{*}+u_{j, i}^{*}\right)+\left(\frac{v}{1-2 v}\right) u_{k, k}^{*} \delta_{i j}\right]_{j}=0
$$

where $p$ is the local hydrodynamic pressure, $U$ is the relative sliding velocity, $u_{i}^{*}$ represents the components of the elastomer displacement vector distribution, and $\delta_{i j}$ is the Kronecker delta. The index notation of the general form $u_{i, j}$ denotes partial derivative of $u_{i}$ with respect to $x_{j}$, and a repeated index denotes summation (e.g., $u_{k, k}^{*}=u_{1,1}^{*}+u_{2,2}^{*}+u_{3,3}^{*}$ ). In order to evaluate the load carrying capacity and the viscous friction force, a simultaneous solution of Eqs. (26) and (27) is required [24].

The dimensionless form of the problem parameters consists of normalizing all length dimensions by $r_{\mathrm{p}}$ (see Ref. [23]) in addition to

$$
\varepsilon=\frac{h_{\mathrm{p}}}{2 r_{\mathrm{p}}} ; \quad P=\frac{\left(p-p_{\mathrm{a}}\right) r_{\mathrm{p}}}{6 \mu U} ; E=\frac{E^{*} r_{\mathrm{p}}}{6 \mu U}
$$

where $p_{\mathrm{a}}$ is the ambient pressure and $E^{*}$ is the Young's modulus of the elastomer.

The dimensionless forms of the local film thickness (Eq. (25)), the Reynolds equation (Eq. (26)), and the elasticity equations (Eq. (27)) are given as

$$
\begin{gathered}
H\left(X_{1}, X_{3}\right)=-C+H_{0}\left(X_{1}, X_{3}\right)+\Delta\left(X_{1}, X_{3}\right) \\
\frac{\partial}{\partial X_{1}}\left(H^{3} \frac{\partial P}{\partial X_{1}}\right)+\frac{\partial}{\partial X_{3}}\left(H^{3} \frac{\partial P}{\partial X_{3}}\right)=\frac{\partial H}{\partial X_{3}} \\
{\left[\frac{1}{2}\left(u_{i, j}+u_{j, i}\right)+\left(\frac{v}{1-2 v}\right) u_{k, k} \delta_{i j}\right]_{j}=0}
\end{gathered}
$$

Because of periodicity of the surface texturing in the circumferential direction (see Fig. 13(c)), it is sufficient to consider the pressure distribution within just one single dimples column with its proper boundary conditions (see Fig. 13(d)).

The deformations of the elastomer sleeve are also periodic in the circumferential direction $x_{3}$ with the same period of $2 r_{1}$ as the hydrodynamic pressure. Hence, it is sufficient to solve Eq. (31) for a single elastomer slice of width $2 r_{1}$ (shown in Fig. 15(a)) that is associated with the single column of dimples shown in Fig. 13(d). The boundary conditions for Eq. (31) relate the dimensionless local shear and normal stresses, respectively, to the dimensionless elastic displacements 


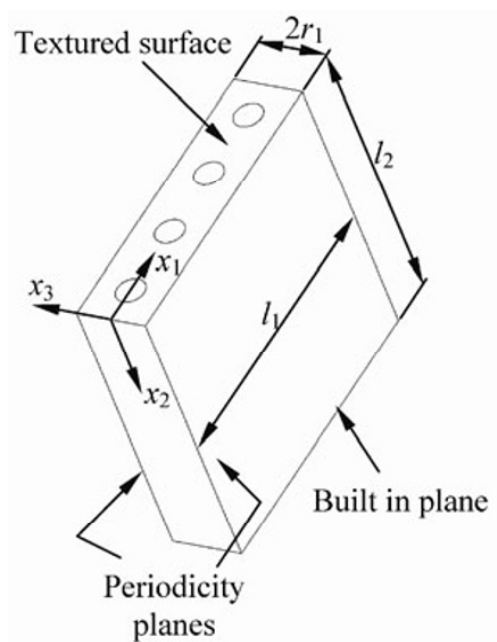

(a)

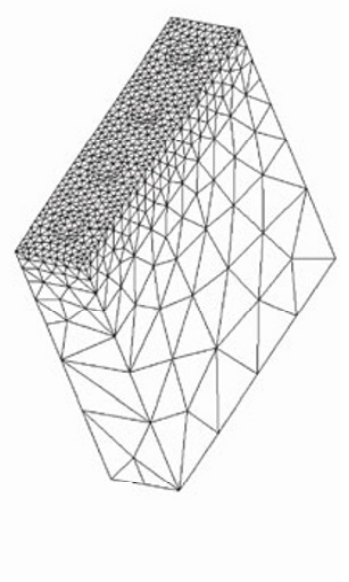

(b)
Fig. 15 Elastomer model: (a) A single elastomer slice (associated with a column of dimples) and its boundary conditions; (b) the finite element mesh of the elastomer slice [24].

(see Ref. [23]) at the inner surface of the elastomer sleeve. These shear and normal stresses are equal to the Couette viscous shear and the hydrodynamic pressures in the fluid, respectively.

The numerical procedure for the simultaneous solution of the Reynolds equation and the elasticity equations consists of the following steps: An initial guess of the film thickness distribution is made and the Reynolds equation Eq. (30) with its proper boundary conditions is solved by a non-uniform grid finite difference method (sees Ref. [8] and Section 3.1 above). This provides a first approximation of the pressure and shear stress distributions, which are used as boundary conditions at the inner surface of the elastomer sleeve.

The elastomer deformations are calculated from Eq. (31) and its boundary conditions (see Ref. [24]) by finite element commercial software ANSYS with higher order 3-D 10-node tetrahedral solid element and non-uniform mesh as shown in Fig. 15(b). The finite element mesh is finest at the textured surface and becomes coarser towards the built-in plane. Local fluid pressure and shear stress are applied at element nodes by using surface elements. The finite element solution provides the deflections of the elastomer and the dimensionless variation in the local fluid film thickness, $\Delta\left(X_{1}, X_{3}\right)$, which in turn are used to correct the fluid film thickness distribution by Eq. (29). The new film thickness distribution is returned to the Reynolds equation and this iterative process is repeated until a desired convergence is achieved.

The dimensionless load carrying capacity, $W$, and friction force, $F_{\mathrm{f}}$, are obtained by:

$$
\begin{gathered}
W=\frac{1}{A} \iint_{A} P\left(X_{1}, X_{3}\right) \mathrm{d} X_{1} \mathrm{~d} X_{3}=\frac{r_{\mathrm{p}}}{6 \mu U} w \\
F_{\mathrm{f}}=\frac{1}{A} \iint_{A} \frac{\mathrm{d} X_{1} \mathrm{~d} X_{3}}{6 H}=\frac{r_{\mathrm{p}}}{6 \mu U} f_{\mathrm{f}}
\end{gathered}
$$

where $A=2 R_{1} L_{1}$ is the dimensionless area of a single dimples column and $w$ and $f_{\mathrm{f}}$ are the dimensional average pressure and shear on the elastomer slice inner surface, respectively. It should be noted here that in textured conformal surfaces, the pressure gradient effect on the friction force is much smaller compared to that of the viscous shear. Hence, the former can be safely neglected. Finally, the coefficient of friction is defined as the ratio of the friction force over the load capacity:

$$
\eta=\frac{f_{\mathrm{f}}}{w}=\frac{F_{\mathrm{f}}}{W}
$$

The model dimensionless parameters required to investigate the load capacity and friction are the following: The operating conditions are represented by the SEHL stiffness index, $E=E^{*} r_{\mathrm{p}} /(6 \mu U)$, the interference fit, $C=c / r_{\mathrm{p}}$, and the pressure differential $P_{\mathrm{s}}$. The elastomer geometry is given by the sleeve dimensions $L_{1}=l_{1} / r_{\mathrm{p}}$ and $L_{2}=l_{2} / r_{\mathrm{p}}$. The texturing parameters include the dimple aspect ratio, $\varepsilon=h_{\mathrm{p}} / 2 r_{\mathrm{p}}$ and the area density, $S_{\mathrm{p}}$.

Differently from all the previous applications that are characterized by rigid mating surfaces, in SEHL there is only a weak optimum of the texturing parameters regarding the load capacity. This can be explained by the opposite effects of hydrodynamic pressure and fluid film thickness on the load capacity. An effective texturing which would increase the hydrodynamic pressure also increases the elastomer radial deformation, which increases the film thickness and hence, offsets the effect of the texturing on the load capacity. However, this increase of the film thickness has a beneficial effect on reducing the viscous shear and therefore, it reduces the friction force and the coefficient of friction. 


\section{The validity of the Reynolds equation for textured surfaces}

Although the theoretical models based on solving the Reynolds equation showed good agreement with experimental results (see Refs. [8, 13, 15, 19]), for example, in Fig. 16 taken from Ref. [15], it was argued on several occasions, e.g., Ref. [27] that the Reynolds equation may not be valid when applied to textured features that have large aspect ratio (the ratio of depth over diameter or width) and that the full NavierStokes (NS) equations should be employed. In order to clarify this issue, both the full NS equations and the Reynolds equation were solved for the case of a compressible fluid at no sliding but with a pressure differential to simulate a hydrostatic gas seal [28]. A comparison between the two solution methods illustrated that in spite of potential large differences in local pressures the differences in load carrying capacity are small for realistic geometrical parameters of LST. Hence, the Reynolds equation can be safely used for most LST applications.

It should be noted here that, as discussed in Ref. [6], surface texturing was also attempted in non-parallel sliding with full hydrodynamic lubrication applications such as thrust bearings and journal bearings. However, in these cases the texturing is beneficial only when the global film convergence is small enough as is shown, for example, in Ref. [29] for textured magnetic recording sliders (see Figs. 17 and 18).

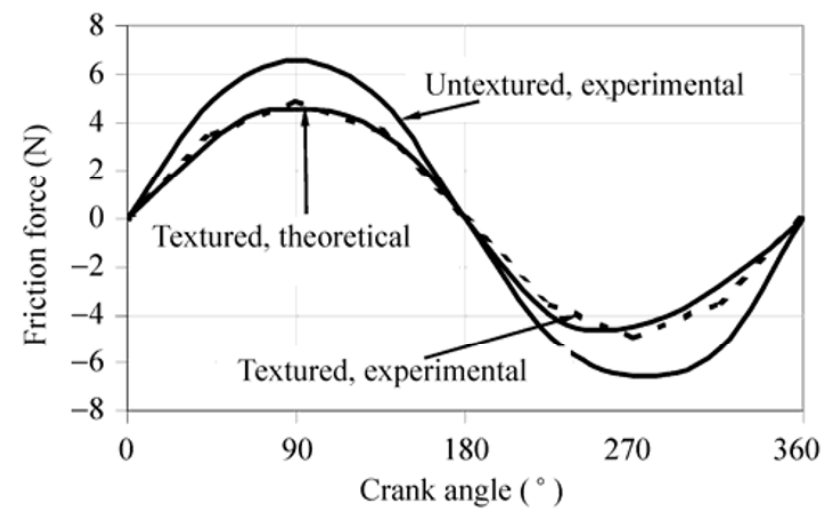

Fig. 16 Correlation between experimental and theoretical results of friction-time variation for simulated textured piston rings at $1000 \mathrm{rpm}$ under full lubrication condition [15].

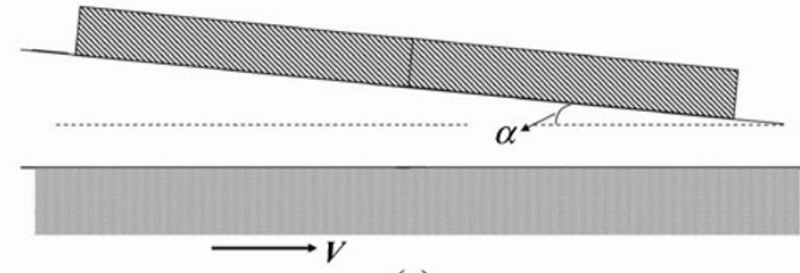

(a)
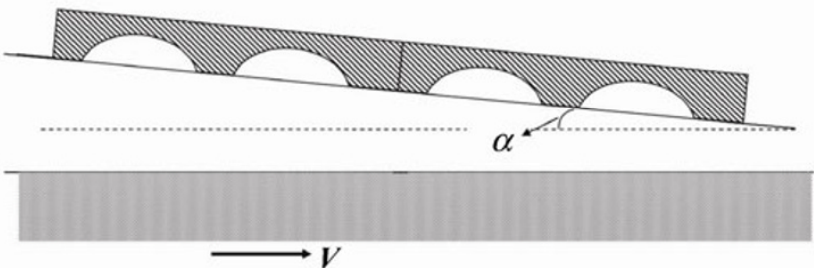

(b)

Fig. 17 Schematic of (a) plane inclined slider bearing and (b) textured slider with pitch angle $\alpha$ [29].

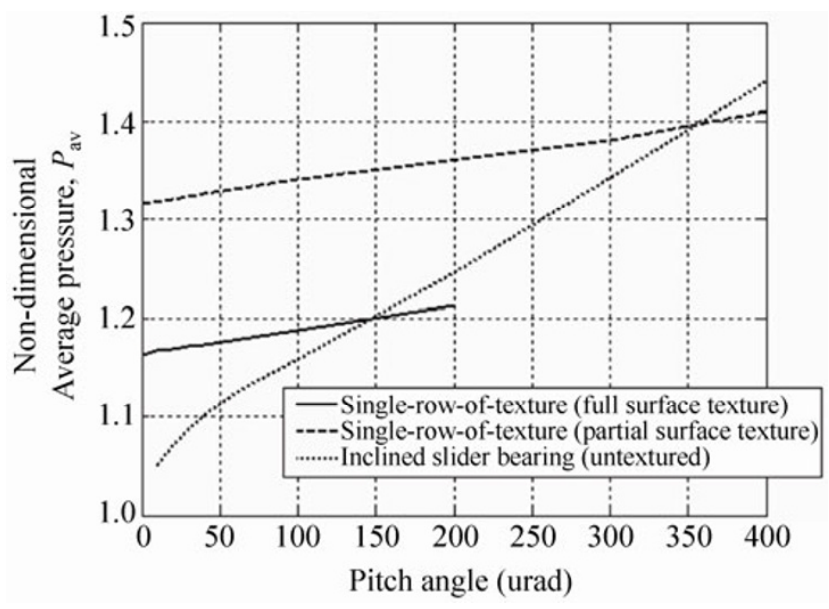

Fig. 18 Non-dimensional average pressure as a function of pitch angle $\alpha$ for the un-textured and textured sliders of Fig. 17, showing that surface texturing is beneficial only at small pitch angles [29].

In yet another non-conformal contact application such as ball bearings it was found that surface texturing can be beneficial only with very shallow dimples [30]. These limitations underline again the most beneficial use of surface texturing in parallel surfaces hydrodynamic and hydrostatic applications.

\section{Optimization}

In order to fully benefit from surface texturing a proper optimization of the geometrical parameters must be performed in accordance with the application in hand. This includes the aspect ratio of the dimples, their 
area density and, in cases of partial surface texturing, the textured portion. The preferred and most efficient way to optimize surface texturing is by parametric analysis in a theoretical model. This was done in several models for bearings [18, 29], various seals [8, $11,13,23,24,31]$, piston rings [14, 26] and magnetic recording tapes [32] to obtain maximum load capacity, minimum friction, maximum film stiffness and minimum leakage. Many experimental studies attempting optimization by trial and error approach can also be found in the literature (see Ref. [6]). These include, for example, different texturing geometries and dimple shapes like squares, triangles, ellipses, grooves etc. Unfortunately, quite often wrong conclusions are arrived at in these studies due to insufficient experimental data. Another typical mistake, which should be avoided in optimizing surface texturing, concerns a comparison of different geometrical shapes based on a certain selected value for a common parameter such as dimple size or area density, for example. Here too, wrong conclusions are usually made regarding the optimum shape for best performance. This is because the certain selected value for the common parameter may be far from being the optimum one for some of the different shapes. The correct procedure for finding an optimum texturing among different shapes is first to optimize each shape individually in terms of its own parameters and only then compare the individual optimums of the different shapes to find the ultimate one. Such a procedure is described in Ref. [17] where an optimum conventional un-textured barrel-shaped piston ring was compared with an optimum surface textured cylindrical piston ring in a firing diesel engine resulting in about $4 \%$ improvement in fuel consumption with the optimum flat face textured ring. In previous studies, when the texturing was applied to the conventional barrelshaped piston ring no difference was observed between the textured and un-textured cases, leading to the wrong conclusion that surface texturing has no benefit in piston rings.

\section{Summary}

Surface texturing, and more specifically LST technology, has great potential in improving tribological performance of various mechanical components over a wide range of different operating conditions. The micro-dimples produced on the surface can act as micro-hydrodynamic bearings in cases of full or mixed lubrication with either incompressible or compressible lubricants.

Surface texturing is most beneficial in cases of parallel sliding with full fluid films. In these cases the effect of texturing geometry on the tribological performance can be easily modeled and optimized for best required performance. Such optimization can provide substantial reduction in friction losses and result in much desired energy savings. In non-parallel/ non-conformal lubricated contacts the benefit of surface texturing is rather limited and may even become detrimental $[29,33]$.

The present invited review naturally focuses mainly on the work done by the author's group at the Technion. Work by others on theoretical, numerical and experimental aspects of textured surface lubrication can also be found in the relevant literature. Most of this work was cited and described in many of the References below and more specifically in Ref. [34].

Open Access: This article is distributed under the terms of the Creative Commons Attribution License which permits any use, distribution, and reproduction in any medium, provided the original author(s) and source are credited.

\section{References}

[1] Etsion I, Burstein L. A model for mechanical seals with regular microsurface structure. Tribol T 39: 677-683 (1996)

[2] Etsion I, Halperin G, Greenberg Y. Increasing mechanical seal life with laser-textured seal faces. In Proc. 15th Int. Conf. on Fluid Sealing, Maastricht, Netherlands, 1997: 3-11.

[3] Geiger M, Roth S, Becker W. Influence of laser-produced microstructures on the tribological behavior of ceramics. Surf Coat Tech 100-101: 17-22 (1998)

[4] Geiger M, Popp U, Engel U. Excimer laser micro texturing of cold forging tool surface-Influence on tool life. CIRP Ann-Manuf Techn 51: 231-234 (2002)

[5] Etsion I. State of the art in laser surface texturing. J Tribol Trans ASME 127: 248-253 (2005)

[6] Etsion I. Laser surface texturing and applications. In Recent 
Developments in Wear Prevention, Friction and Lubrication. Nikas G K, Ed. Kerala (India): Research Signpost, 2010: 137-158.

[7] Etsion I. Surface texturing for in-cylinder friction reduction. In Tribology and Dynamics of Engine and Powertrain: Fundamentals, Applications and Future Trends. Rahnejat H, Ed. New Delhi (India): Woodhead Publishing Ltd., 2010: 458-469.

[8] Etsion I, Kligerman Y, Halperin G. Analytical and experimental investigation of laser-textured mechanical seal faces. Tribol T 42: 511-516 (1999)

[9] Etsion I. Improving tribological performance of mechanical seals by laser surface texturing. In Proc. $17^{\text {th }}$ Int. Pump Users Symposium, 2000: 17-22.

[10] Yu X Q, He S, Cai R L. Frictional characteristics of mechanical seals with a laser-textured seal face. $J$ Mater Process Tech 129: 463-466 (2002)

[11] Kligerman Y, Etsion I. Analysis of the hydrodynamic effects in a surface textured circumferential gas seal. Tribol T 44: 472-478 (2001)

[12] McNikel A, Etsion I. Near-contact laser surface textured dry gas seals. J Tribol Trans ASME 126: 788-794 (2004)

[13] Etsion I, Halperin G. A laser surface textured hydrostatic mechanical seal. Tribol T 45: 430-434 (2002)

[14] Ronen A, Etsion I, Kligerman Y. Friction-reducing surface texturing in reciprocating automotive components. Tribol $T$ 44: 359-366 (2001)

[15] Ryk G, Kligerman Y, Etsion I. Experimental investigation of laser surface texturing for reciprocating automotive components. Tribol T 45: 444-449 (2002)

[16] Golloch R, Merker G P, Kessen U, Brinkmann S. Benefits of laser-structured cylinder liners for internal combustion engines. In Proc. $14^{\text {th }}$ International Colloquium Tribology, Esslingen, Germany, 2004: 321-328.

[17] Etsion I, Sher E. Improving fuel efficiency with laser surface textured piston rings. Tribol Int 42: 542-547 (2009)

[18] Brizmer V, Kligerman Y, Etsion I. A laser surface textured parallel thrust bearing. Tribol T 46: 397-403 (2003)

[19] Etsion I, Halperin G, Brizmer V, Kligerman Y. Experimental investigation of laser surface textured parallel thrust bearings. Tribol Lett 17: 295-300 (2004)

[20] Hayashi H, Taylor C M. A determination of cavitation interfaces in fluid film bearings using finite element analysis.
J Mech Eng Sci 22: 277-285 (1980)

[21] Elrod H G. A cavitation algorithm. J Lubr Technol Trans ASME 103: 350-354 (1981)

[22] Gerald C F, Wheately P O. Applied Numerical Analysis, 5th Edition. New York: Addison-Wesley Publishing Co., 1994.

[23] Shinkarenko A, Kligerman Y, Etsion I. The effect of elastomer surface texturing in soft elasto-hydrodynamic lubrication. Tribol Lett 36: 95-103 (2009)

[24] Shinkarenko A, Kligerman Y, Etsion I. Theoretical analysis of surface textured elastomer sleeve in lubricated rotary sliding. Tribol T 53: 376-385 (2010)

[25] Reddy J N. An Introduction to the Finite Element Method. New York: McGraw-Hill Inc., 1993.

[26] Kligerman Y, Etsion I, Shinkarenko A. Improving tribological performance of piston rings by partial surface texturing. $J$ Tribol Trans ASME 127: 632-638 (2005)

[27] Sahlin F, Glavatskih S B, Almkvist T, Larsson R. Twodimensional CFD analysis of micro-patterned surfaces in hydrodynamic lubrication. J Tribol Trans ASME 127: 96-102 (2005)

[28] Feldman Y, Kligerman Y, Etsion I, Haber S. The validity of the Reynolds equation in modeling hydrostatic effects in gas lubricated textured parallel surfaces. J Tribol Trans ASME 128: $345-350$ (2006)

[29] Murthy A N, Etsion I, Talke F E. Analysis of textured air bearing sliders with rarefaction effects. Tribol Lett 28: 251-261 (2007)

[30] Mourier L, Mazuyer D, Lubrecht A A, Donnet C. Transient increase of film thickness in micro-textured EHL contacts. Tribol Int 39: 1745-1756 (2006)

[31] Feldman Y, Kligerman Y, Etsion I. Stiffness and efficiency optimization of a hydrostatic laser surface textured gas seal. J Tribol Trans ASME 127: 407-410 (2007)

[32] Raeymaekers B, Etsion I, Talke F E. A model for magnetic tape/guide friction reduction by laser surface texturing. Tribol Lett 28: 9-17 (2007)

[33] Kovalchenko A, Ajayi O, Erdemir A, Fenske G. Friction and wear behavior of laser textured surface under lubricated initial point contact. Wear 271: 1719-1725 (2011)

[34] Etsion I. Surface texturing. In Handbook of Lubrication and Tribology, Volume II: Theory and Design, Second Edition. Bruce R W, Ed. Boca Raton (USA): CRC Press, 2012. 


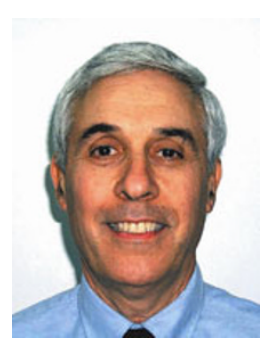

Izhak ETSION. He received his $\mathrm{PhD}$ degree in 1974 from Technion-Israel Institute of Technology. Since then he was a faculty at the Department of Mechanical Engineering where he is currently a Professor Emeritus. He is the author and co-author, together with his many graduate students, of over 190 technical publications on various aspects of tribology. He holds 15 patents and is the founder of Surface Technologies Ltd. that developed the laser surface texturing (LST) technology for friction and wear reduction. His research interests are in hydrodynamic lubrication, surface texturing, contact mechanics, nano-tribology and bio-tribology. $\mathrm{He}$ is a frequent invited scientist to academic institutions worldwide, among the more recent ones are Northeastern University and UC San Diego in the US, Cambridge University in the UK, Tsinghua University in China, UWA in Australia, the Indian Institute of Science in India and the University of Sao Paulo in Brazil.

In addition to his academic activities, he also served the tribology community as founder of the Israel Tribology Society, Associate Editor of Journal of Tribology published by ASME, Secretary of the ASME Tribology Division Executive Committee, Chairman of ASME Tribology Division Nomination and Oversight Committee, and Chair of the STLE Fellows Committee.

He is a Member of the Israel Tribology Society, Fellow of the ASME and Fellow of the STLE. He is also the recipient of the STLE 2005 International Award. 